neuromuscular conditions the North Star Ambulatory Assessment is valid, reliable and practical as a functional assessment, and includes activities necessary to remain functionally ambulant. With regards to MPS, searches did not reveal specific MSK tests, but evidence suggests that skeletal malformations and joint problems are the most frequently presenting signs. pGALS performs well to identify abnormal joints with restriction within an MPS group.

Conclusion This review supports the development of a 'pGALSplus' toolkit to facilitate identification of CYP with potentially serious disease. pGALSplus will be targeted at community-based clinicians and will likely include physical examination, questionnaire(s) and appropriate adjuncts.

\section{G315(P) ABSTRACT WITHDRAWN}

\section{G316(P) AUTISM AND CSLE (CHILDHOOD ONSET SYSTEMIC LUPUS ERYTHEMATOSUS) DIAGNOSTIC DILEMMA: A CASE OF RARE COMBINATIONS}

C Devanahalli Nagaraj, J Harris, S Arani. Paediatrics, Leicester Royal Infirmary, Leicester, UK

\subsection{6/archdischild-2020-rcpch.273}

Case We present a case of a 7-year-old girl with severe autism presenting with facial swelling and bilateral cervical lymphadenopathy initially thought to be lymphoma but later diagnosed as systemic lupus with nephritis and neuro-lupus. Her initial course was stormy needing admission to intensive care unit and referral to oncology centre for work up of lymphoma.

This child had no significant hospitalisations in the past. Her initial blood results and CT (Computerised tomography) chest suggestive of lymphoma with tumor lysis. She was transferred to a local paediatric oncology centre for lymph node and bone marrow testing which were both negative. She developed focal seizure secondary to hypertension and PRES (Posterior reversible encephalopathy), needing intubation and transferred to intensive care unit for 48 hours. Magnetic resonance (MRI) imaging brain showed signs consisted with cerebral lupus. Autoimmune screen strongly positive for antibodies ANA (Antinuclear), ds DNA (double stranded deoxyribonucleic acid), Anti-Ro, Anti-Smith, RNP (Ribonucleoprotein), RNP70, low C3 (complement) and C4 in keeping with SLE.

Course She was treated with intravenous (IV) pulsed methylprednisolone for 3 days, followed by oral prednisolone, IV cyclophosphamide and later switched to Mycophenolate (MMF) therapy with which she made good recovery. Renal biopsy was not performed as the child remained hypertensive despite being on antihypertensive medications. Other complications included ascites, pleural effusion and pericardial effusion which was managed conservatively. She stayed in the hospital for 2 months with good neurological recovery back to her previous status.

At 3 months - She remains on immunosuppressive medication i.e. Mycophenolate and tapering doses of oral prednisolone with good clinical response. MRI head shows significant improvement in comparison to her previous images, complete resolution of the pericardial effusion and urine examination no evidence of proteinuria or haematuria.
Conclusion cSLE (Childhood Systemic Lupus Erythematosus) with multi-system involvement in an autistic child can be difficult to diagnose and manage. The case highlights that cSLE is a disease of thousand faces with varied clinical presentations and the need for high index of suspicion. This case also highlights the role of multidisciplinary teams in the management of such complex cases.

\section{G317(P) ABSTRACT WITHDRAWN}

\section{G318(P) BRITISH SOCIETY OF PAEDIATRIC AND ADOLESCENT RHEUMATOLOGY (BSPAR) STANDARDS OF CARE FOR UVEITIS SCREENING IN CHILDREN WITH JUVENILE IDIOPATHIC ARTHRITIS (JIA) - COMPLIANCE IN A SINGLE CENTRE IN SOUTHERN IRELAND}

${ }^{1} \mathrm{D}$ O' Sullivan, 'C Foley, ${ }^{1} \mathrm{DA}$ Deely, 'S Felsenstein. 'Department of Paediatrics, Cork University Hospital, Cork, Ireland; ${ }^{2}$ Department of Rheumatology, Children's Health Ireland at Crumlin, Dublin, Ireland

\subsection{6/archdischild-2020-rcpch.274}

Background Uveitis is an extra-articular manifestation of Juvenile Idiopathic Arthritis (JIA) that affects up to $30 \%$ of patients. Presentation is classically insidious and may cause blindness. It is essential that regular slit-lamp examination is performed in all cases of JIA. BSPAR/Royal College of Ophthalmology (RCO) Uveitis Screening principles recommend following JIA diagnosis, slit-lamp examination should occur;

- Immediately and no later than 6 weeks from referral (recommendation 1)

- Every 2 months, from onset of arthritis, for 6 months (recommendation 2)

- Every 3-4 months thereafter (recommendation 3)

Aims To describe compliance with BSPAR/RCO guidelines in newly diagnosed cases of JIA.

Methodology A retrospective chart review of children with JIA attending our Centre was performed to ascertain compliance with BSPAR/RCO guidelines.

Results 28 children with a diagnosis of JIA attend our Centre (Female $\mathrm{n}=23,82 \%$ ). Nine patients were ANA positive $(28 \%)$. Classification of patients identified 39\% $(n=11)$ had polyarticular JIA, 22\% $(n=6)$ had Oligoarticular JIA, $14 \%(n=4)$ had systemic onset JIA, 14\% $(n=4)$ had Psoriatic JIA and $11 \% \quad(n=3)$ had enthesitis-related arthritis. Table 1 details results of compliance with BSPAR/RCO guidelines.

\begin{tabular}{lllll}
$\begin{array}{l}\text { Abstract G318(P) Table 1 } \\
\text { Compliance }\end{array}$ & Yes & No & Not documented & Not applicable* \\
n (\%) & & & & \\
\hline Recommendation 1 & $9(32 \%)$ & $16(57 \%)$ & $3(11 \%)$ & - \\
Recommendation 2 & $5(18 \%)$ & $18(64 \%)$ & $3(11 \%)$ & $2(7 \%)$ \\
Recommendation 3 & $3-4$ monthly & $>4$ monthly & $3(10.5 \%)$ & $3(10.5 \%)$ \\
& $8(29 \%)$ & $14(50 \%)$ & &
\end{tabular}

*Not attending service for period to be assessed i.e. relatively new JIA diagnosis 
Discussion Current practice at our Centre, as judged against the BSPAR/RCO standards of care, is suboptimal. We report a delay in access to an Ophthalmologist for initial slit-lamp examination following JIA diagnosis and follow-up reviews are less frequent than is best practice. This audit has highlighted need for improvement in the provision of care for our patients with JIA and we intend to utilise this data as the basis for future quality improvement initiatives.

\section{G319(P) DRAMATIC RESPONSE OF FAMILIAL MAJEED SYNDROME TO INTERLEUKIN-1 ANTAGONIST THERAPY: CASE REPORT}

'B Almoosawi, ${ }^{2} Z$ Almusawi, ${ }^{2} \mathrm{~W}$ Madan, ${ }^{3} \mathrm{~S}$ Alwadaei, ${ }^{4} \mathrm{H}$ Naser. 'General Paediatrics, RCPCH, Liverpool, UK; ${ }^{2}$ SPaediatric, Salmaniya Medical Complex, Manama, Bahrain; ${ }^{3}$ Neurology, RCP, Birmingham, UK; ${ }^{4}$ Radiology, Salmaniya Medical Complex, Manama, Bahrain

\subsection{6/archdischild-2020-rcpch.275}

Majeed syndrome (MS) is a rare, autosomal recessive, autoinflammatory disease characterized by recurrent multifocal osteomyelitis, congenital dyserythropoietic anemia, and inflammatory dermatome. In this article, we report the cases of two siblings with MS. Genetic studies of both siblings were obtained and revealed mutations in LPIN2 gene by means of a homozygous single-base pair change in the donor splice site of exon 17 (c. $2327+1 G>C$ ). Both patients underwent different modalities of treatment for MS which involved immunesuppressive and biologic therapies. We observed a significant clinical response to biologic anti-interleukin-1 (IL-1) therapy in our patients. This impressive clinical response indicates the pivotal role of IL-1 in MS pathogenesis. There are limited data on the use of anti-IL-1 therapy in treating MS due to the rarity of the condition. Anti-IL-1 therapy should be considered as a promising treatment for this disease.

\section{G320(P) ADENOSINE DEAMINASE 2 DEFICIENCY WITH A NOVEL VARIANT OF CECR1 GENE MUTATION: RESPONDING TO TUMOR NECROSIS FACTOR ANTAGONIST THERAPY}

B Almoosawi, Z Almusawi, ${ }^{3}$ Hiba Omar, ${ }^{4}$ B Maryam. 'Liverpool Women's Hospital, NHS Foundation Trust, Liverpool, UK; ${ }^{2}$ Salmaniya Medical Complex, Ministry of Health, Bahrain; ${ }^{3}$ King Hamad University Hospital, Bahrain; ${ }^{4}$ Genetics Department, Salmaniya Medical Complex, Ministry of Health, Bahrain

10.1136/archdischild-2020-rcpch.276

Deficiency of Adenosine deaminase 2 (DADA2) syndrome is a chronic, systemic, and inflammatory disorder, characterized by early-onset recurrent strokes, fever, livedo reticularis, and immunodeficiency. We report the case of a 4 -year-old child, a product of consanguineous marriage, who presented with three episodes of hemiparesis within 1 year. She also manifested skin discoloration in the form of livedo reticularis. Workup with magnetic resonance imaging (MRI) of the brain revealed acute infarction in the right aspect of the cerebral peduncle and chronic lacunars infarct in the right thalamus with diffusion restriction. Repeated MRI after 5 months revealed diffuse loss of brain volume. The blood workup showed high inflammatory markers and significantly low adenosine deaminase 2 (ADA2) level. After being on corticosteroid and anticoagulant treatments, she suffered from a recurrent episode of cerebral infarction, after which she was commenced on tumor necrosis factor (TNF)-antagonist therapy in addition to monthly fresh plasma infusion. Thereafter, there was no cerebral insult reported for $>18$ months. The genetic study of the child and her parents revealed a homozygous mutation c. $336 \mathrm{C}>\mathrm{A}$, p. (His112Gln) in the CECR1 gene, and her parents were heterozygous for the same variant. This variant was not previously reported in literature. We would suggest linking this novel variant c. 336C>A, p. (His112Gln) of CECR1 gene mutation with the clinical picture, along with the positive response to TNF-antagonist therapy in the era of ADA2 deficiency syndrome.

\section{British association for paediatric nephrology}

\section{G321 INPATIENT ADMISSION AND ED REATTENDANCE RATES IN A COHORT OF CHILDREN WITH IGA VASCULITIS}

${ }^{1} \mathrm{G}$ Haigh, ${ }^{1,2} \mathrm{~L}$ Oni, ${ }^{3} \mathrm{~S}$ Mulvaney. ${ }^{1}$ Department of Paediatric Nephrology, Alder Hey Children's NHS FT, Liverpool, UK; '2Department of Women's and Children's Health, University of Liverpool, Liverpool, UK; ${ }^{3}$ Department of Emergency Medicine, Alder Hey Children's NHS FT, Liverpool, UK

\subsection{6/archdischild-2020-rcpch.277}

Aim To determine the impact of IgA vasculitis (Henoch Schonlein purpura) on the emergency and acute medical services in a regional paediatric teaching hospital.

Method The case notes of all patients attending Alder Hey Children's Hospital emergency department (ED) and coded as HSP between 1st January 2018 and 31st December 2018 were reviewed retrospectively. Data was collected to establish reasons for admission and reattendance, time between diagnosis and reattendance, and length of inpatient stay on admission.

Results A cohort of 53 patients presented with a new diagnosis of HSP during 2018. 62\% were male, 38\% female. The mean age was 6 years (range 1 to 13 years). 11 patients (21\%) were admitted for symptom management or further investigation. Of these, $4(36 \%)$ were admitted on presentation and $7(64 \%)$ on reattendance. Reasons for admission on presentation included manual blood pressure monitoring (2) and joint pain requiring analgesia (2). All patients were discharged within 24 hours. 14 patients (26\%) reattended ED with problems relating to HSP. Time between diagnosis and reattendance ranged from 1 day to 123 days $($ mean $=9$ days). The primary reasons for reattendance were pain (10/ $14 ; 71 \%)$, worsening/recurring rash $(3 / 14 ; 21 \%)$, and limb swelling $(1 / 14 ; 7 \%)$. Of the 10 patients representing with pain 5 complained of joint pain, 2 of abdominal pain, and 3 of both joint and abdominal pain. $50 \%$ of the reattending patients required admission (joint pain $5 / 7 ; 71 \%$, abdominal pain $2 / 7$; 29\%). All patients requiring admission after reattending with joint pain were admitted for less than 24 hours. The 2 patients with abdominal pain alone were diagnosed with intussusception which resolved with non-operative management and had an inpatient stay of 1 and 3 days respectively.

Conclusion The impact of IgA vasculitis in children extends beyond the potential renal involvement. Patient's experience of pain is a significant factor and leads to a high reattendance/ admission to hospital rate. Ways to improve supporting patients to recover at home are being investigated as specialist intervention was rarely needed. 